\title{
Nonlinear Polarization Manipulation in Optical Fibers
}

\author{
P-Y. Bony ${ }^{1}$, M. Guasoni ${ }^{1}$, M. Gilles ${ }^{1}$, A. Picozzi ${ }^{1}$, S. Pitois ${ }^{1}$, G. Millot ${ }^{1}$, S. Wabnitz ${ }^{2}$ and J. Fatome ${ }^{1}$ \\ 1 - Laboratoire Interdisciplinaire Carnot de Bourgogne, UMR 6303 CNRS - Université Bourgogne Franche-Comté, Dijon, France \\ 2 - Department of Information Engineering, Università di Brescia, Via Branze 38, 25123 Brescia, Italy
}

\begin{abstract}
We describe a phenomenon of self-organization of the light state-of-polarization in optical fibers based on a nonlinear cross-polarization interaction between an incident signal and its backward replica. Several proof-of-principles for telecom applications are reported.

OCIS codes: (060.0060) Fiber optics and optical communications; (060.4370) Nonlinear optics, fibers; (250.4745) Optical processing devices.
\end{abstract}

\section{Introduction}

In many fields of photonics, in particular in all-fiber based systems, the light state-of-polarization (SOP) remains one of the most elusive variables, which is still difficult to predict and control [1]. Indeed, despite the impressive progress in the manufacturing process of modern Telecom optical fibers, the residual birefringence combined with environmental surrounding stress and variations like bending, squeezing, vibrations or temperature fluctuations make the polarization of light unpredictable after only a few hundreds of meters of propagation. In this context, the all-optical repolarization of an incoming signal without loss of energy becomes of a very high interest. To this purpose and to surpass standard polarizers which vanish up to $50 \%$ of an incoming unpolarized light, several techniques have emerged in the literature in order to develop a "universal polarizer" which could lead to a complete repolarization of light with $100 \%$ efficiency [2-6]. This phenomenon of polarization pulling has been the subject of several works, involving the Raman and Brillouin scatterings [2-3], parametric amplification [4] as well as a counter-propagating four-wave mixing process [5]. The common issue of these above devices is that a fully polarized pump beam is used as polarization reference for the incoming signal and therefore has to be perfectly stabilized in turn. To overcome this issue, here we explore a different approach and exploit the unique capability for a light beam to self-organize its own state-of-polarization, upon nonlinear propagation in optical fibers, into universal and environmentally robust states, namely right and left circular polarizations [6]. This nonlinear device, called Omnipolarizer is able to self-organize the light SOP without any need of additional control and provides a wide range of dynamics, which allowed us to experimentally demonstrate several all-optical functions for Telecom applications such as digital polarization-beam-splitter, ideal polarizer, optical flip-flop memory as well as all-optical polarization switch and all-optical scrambler [6-8].

\section{Principle}

Basically, as illustrated in Fig. 1, the Omnipolarizer consists in a single segment of optical fiber (typically $5 \mathrm{~km}$ ), in which the arbitrary polarized incoming signal nonlinearly interacts through a four-wave-mixing process with its own counter-propagating replica generated by means of a back-reflective element inserted at the fiber end. Depending on the reflective coefficient $\mathrm{R}$ (which can be even greater than 1 when the backward signal is amplified into a reflective-loop), the Omnipolarizer may operate in three distinct regimes: A digital polarization beam splitter (PBS) for $\mathrm{R}<1$, a nonlinear polarizer for $\mathrm{R}$ slightly larger than 1 as well as an all-fiber polarization scrambler for $\mathrm{R}>>1$.

\section{Experimental setup}

The experimental setup is basically illustrated by Fig. 1. An initial 40-Gbit/s On/Off Keying signal centered on $1564 \mathrm{~nm}$ is first generated and polarization scrambled. This incoming signal is then amplified thanks to an Erbium doped fiber amplifier (EDFA) until $27 \mathrm{dBm}$ before injection into a 6.2-km long non-zero dispersion fiber (NZ-DSF) characterized by a normally dispersive regime $\mathrm{D}=-1.5 \mathrm{ps} / \mathrm{nm} / \mathrm{km}$ at $1550 \mathrm{~nm}$ and a Kerr coefficient $\gamma=1.7 \mathrm{~W}^{-1} \mathrm{~km}^{-1}$. This incident signal then nonlinearly interacts with its own counter-propagating replica generated at the fiber output by means of a reflective loop setup consisting in an optical circulator and a second EDFA. A 90/10 coupler is also added within the back-reflective loop so as to evict the stabilized signal for analysis.

\section{Experimental results}

The experimental results obtained for the two first operating regimes are summarized in Fig. 1. Depending on the back-reflective coefficient R, basically the gain of the second EDFA, two distinct regimes are obtained. When $\mathrm{R}$ is slightly larger than 1 (i.e., the back-reflected light is then amplified up to $28 \mathrm{dBm}$ ), any arbitrary input signal SOP converges towards a fixed and unique SOP. In this configuration the Omnipolarizer acts as an ideal nonlinear 
polarizer which enables to reach a degree-of-polarization close to unity. This behavior is even more striking in the time domain. Indeed, at the input of the device, because of the polarization scrambling process, after detection of the signal beyond a polarizer, all the polarization fluctuations are transferred into the time domain leading to a complete closure of the 40-Gbit/s eye-diagram and loss of the data. At the output of the Omnipolarizer, because of the selfpolarization effect, and despite the initial scrambling process, the resulting eye-diagram remains completely opened beyond the polarizer, indicating that the self-polarization process operates in full strength.

A second optical function can be achieved when the back-reflection coefficient is now below unity, here $\mathrm{R}=0.6$. In this case, any arbitrary input SOP is digitally attracted towards either the right or left circular SOP depending exclusively on the sign of its initial ellipticity. Two small areas are clearly visible on the output Poincaré sphere corresponding to the north (right handed SOP) and south poles (left-handed). The device becomes indeed bistable and its output polarization is characterized by a phenomenon of hysteresis [7]. As a result, we can obtain a discrete polarization beam splitter which can for instance efficiently route data packets on one or the other orthogonal axis with an excellent extinction of the opposite component (larger than $20 \mathrm{~dB}$ ).

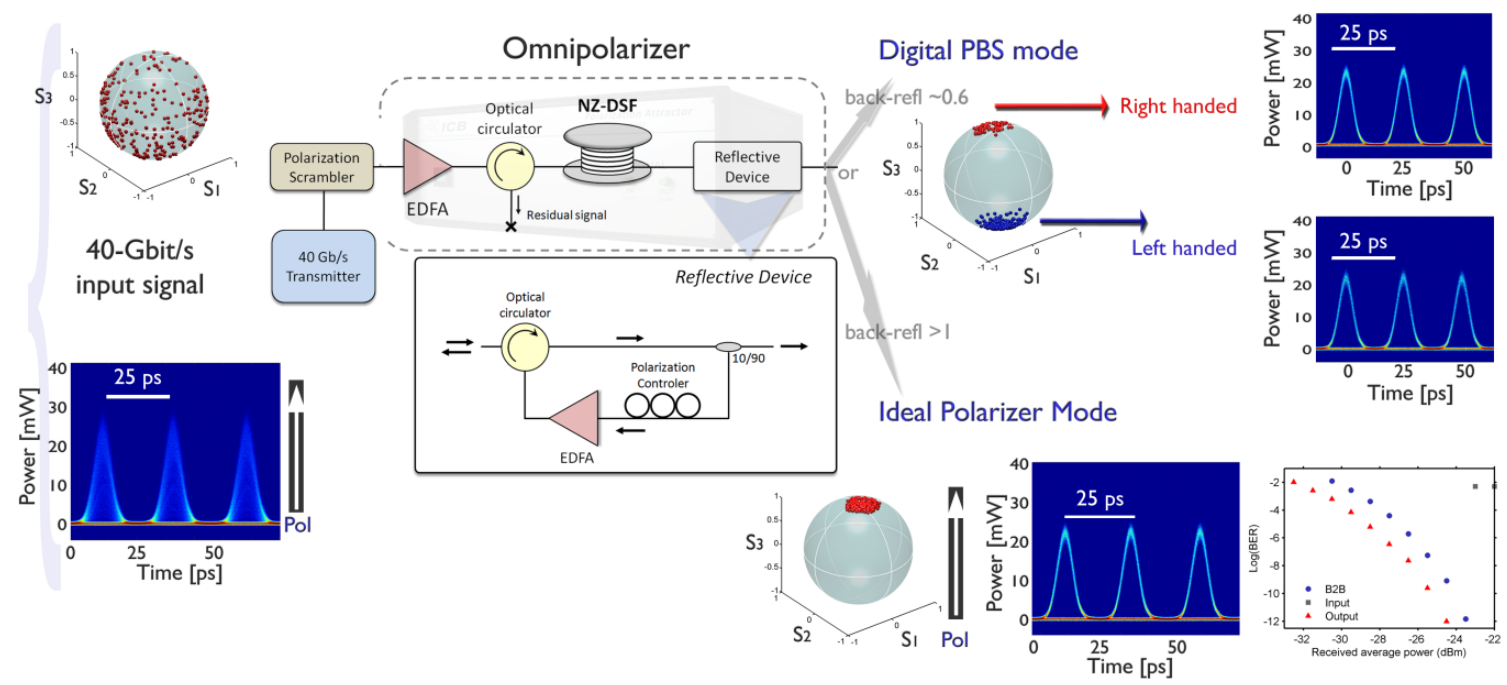

Fig. 1. Experimental results obtained for the Omnipolarizer for an input 40-Gbit/s signal power of $27 \mathrm{dBm}$. Depending on the back-reflected power, the device can act in two operation modes: digital polarization beam splitter (PBS) or ideal polarizer.

In conclusion, we report the experimental observation of a self-organization effect of light state-of-polarization in standard optical fibers. This device, called Omnipolarizer, is based on a nonlinear cross-polarization interaction between a signal beam and its feedback replica generated at the fiber end thanks to a reflective element. The complex and rich dynamics of this system offers a wide range of optical functions such as repolarization of an incident signal, digital beam splitter, all-fiber scrambler, flip-flop memory as well as polarization-based data router.

\section{References}

[1] M. Martinelli, P. Martelli, and S. M. Pietralunga, "Polarization stabilization in optical communications systems," J. Lightw. Technol. 24, 4172-4183 (2006).

[2] M. Martinelli, M. Cirigliano, M. Ferrario, L. Marazzi, and P. Martelli, "Evidence of Raman-induced polarization pulling," Opt. Express 17, 947-955 (2009).

[3] A. Zadok, E. Zilka, A. Eyal, L. Thévenaz, and M. Tur, "Vector analysis of stimulated Brillouin scattering amplification in standard singlemode fibers," Opt. Express 16, 21692-21707 (2008).

[4] B. Stiller, P. Morin, D. M. Nguyen, J. Fatome, S. Pitois, E. Lantz, H. Maillotte, C. R. Menyuk, and T. Sylvestre, "Demonstration of polarization pulling using a fiber-optic parametric amplifier," Opt. Express 20, 27248-27253 (2012).

[5] J. Fatome, S. Pitois, P. Morin, and G. Millot, "Observation of light-by-light polarization control and stabilization in optical fibre for telecommunication applications," Opt. Express 18, 15311-15317 (2010).

[6] J. Fatome, S. Pitois, P. Morin, E. Assemat, D. Sugny, A. Picozzi, H. R. Jauslin, G. Millot, V. V. Kozlov, and S. Wabnitz, "A universal optical all-fiber omnipolarizer," Sci. Rep., 2, 938; DOI:10.1038/srep00938 (2012).

[7] P-Y. Bony, M. Guasoni, E. Assémat, S. Pitois, D. Sugny, A. Picozzi, H. R. Jauslin, and J. Fatome, "Optical flip-flop memory and data packet switching operation based on polarization bistability in a telecommunication optical fiber," J. Opt. Soc. Am. B 30, 2318-2325, (2013).

[8] P-Y. Bony, M. Guasoni, P. Morin, D. Sugny, A. Picozzi, H.R. Jauslin, S. Pitois and J. Fatome, "Temporal spying and concealing process in fibre-optic data transmission systems through polarization bypass," Nat. Commun. 5:4678 (2014). 